\title{
1608+656: A QUADRUPLE-LENS SYSTEM FOUND IN THE CLASS GRAVITATIONAL LENS SURVEY
}

\author{
S. T. Myers, C. D. Fassnacht, S. G. Djorgovski, ${ }^{1}$ R. D. Blandford, K. Matthews, ${ }^{1}$ \\ G. Neugebauer, ${ }^{1}$ T. J. Pearson, A. C. S. Readhead, J. D. Smith, ${ }^{1}$ \\ D. J. ThOMPSON, ${ }^{1}$ AND D. S. WOMBLE ${ }^{1}$ \\ California Institute of Technology, 105-24, Pasadena, CA 91125 \\ I. W. A. Browne, P. N. WILKInSON, AND S. NAIR \\ University of Manchester, Nuffield Radio Astronomy Laboratories, Jodrell Bank, Macclesfield, Cheshire SK11 9DL, England, UK \\ N. Jackson, ${ }^{2}$ I. A. G. SNELlen, AND G. K. Miley \\ Leiden Observatory, Postbus 9513, 2300 RA Leiden, The Netherlands \\ A. G. DE BRUYN ${ }^{3}$ \\ Netherlands Foundation for Research in Astronomy, Postbus 2, 7990 AA Dwingeloo, The Netherlands \\ AND \\ R. T. SCHILIZZI ${ }^{4}$ \\ Joint Institute for VLBI in Europe, Postbus 2, 7990 AA Dwingeloo, The Netherlands \\ Received 1995 March 6; accepted 1995 April 18
}

\begin{abstract}
The first phase of a large gravitational lens survey using the Very Large Array at a wavelength of $3.6 \mathrm{~cm}$ has been completed, yielding images for 3258 radio sources. The Cosmic Lens All-Sky Survey (CLASS) is designed to locate gravitational lens systems consisting of multiply imaged compact components with separations greater than 0.2 . We report here the first discovery of a gravitational lens from the survey: $1608+656$, a quadruply imaged object with a maximum separation of 2". 1 . Images from the Palomar $5 \mathrm{~m}$ and Keck $10 \mathrm{~m}$ telescopes show the lensed images and the lensing galaxy. An optical spectrum obtained with the Palomar $5 \mathrm{~m}$ telescope indicates a redshift of $z=0.6304$ for the lensing galaxy. No conclusive redshift for the lensed object has been determined, although a single strong emission line is found at $9240 \AA$ in the Keck low-resolution imaging spectrograph spectrum. The two most likely identifications for this line are $\mathrm{H} \beta(z=0.90)$ and $\mathrm{Mg}$ II $(z=2.30)$. The preliminary lens model derived from the radio image reproduces the observed configuration and relative fluxes of the images, as well as the position, shape, and orientation of the lensing galaxy. Because a simple mass model is able to fit the observations, we argue that this lens system is promising for determining $H_{0}$.
\end{abstract}

Subject headings: gravitational lensing

\section{INTRODUCTION}

Gravitational lenses provide a unique way of probing the distribution of clumpy matter in the universe and have the potential to give values for the Hubble constant and other cosmological parameters. With this in mind, and building on the success of the Jodrell Bank/VLA Astrometric Survey (JVAS; Patnaik et al. 1992; Browne et al. 1995), we have embarked on a new, much larger VLA survey: the Cosmic Lens All-Sky Survey (CLASS). The ultimate aim of CLASS is to survey $\sim 10^{4}$ flat-spectrum radio sources with the prime motivation of identifying systems suitable for Hubble constant determination based on measurements of time delays. Restricting the sample to flat-spectrum sources preferentially selects objects with compact and variable components well suited to time delay measurements. A total of 3258 targets have been observed in the first phase of CLASS. Based on previous surveys with similar selection criteria (e.g., Patnaik et al. 1992), we expect to find about one lens per 500 sources observed, i.e., about six new lenses should emerge from the

\footnotetext{
1 Also Palomar Observatory, California Institute of Technology.

2 Current address: University of Manchester, Nuffield Radio Astronomy Laboratories, Jodrell Bank, Macclesfield, Cheshire SK11 9DL, England, UK.

3 Also Kapteyn Laboratory.

${ }^{4}$ Also Leiden Observatory.
}

first phase of the survey and, ultimately, about 20 lenses from the survey of the northern hemisphere. CLASS has already yielded two new lens systems: $1600+434$, a double-image system (Jackson et al. 1995), and 1608+656, reported here.

\section{CLASS VLA OBSERVATIONS}

The CLASS observations took place in several sessions during 1994 February, March, and April in the A configuration of the VLA ${ }^{5}$ at a frequency of $8.4 \mathrm{GHz}$. The 3258 sources were selected from the $4.85 \mathrm{GHz}$ Green Bank Survey (87GB; Gregory \& Condon 1991) to have flux densities $\geq 50 \mathrm{mJy}$ and $|b|>10^{\circ}$. At the time of the CLASS VLA observations, we were able to select 683 sources with two-point spectral indices of $\alpha \geq-0.5$ between $4.85 \mathrm{GHz}$ and the $325 \mathrm{MHz}$ Westerbork Northern Sky Survey (de Bruyn et al. 1995). The remaining 2575 sources were selected with $\alpha \geq-0.6$ between $4.85 \mathrm{GHz}$ and the $365 \mathrm{MHz}$ Texas Survey. The lens $1608+656$ was found in the $87 \mathrm{~GB} /$ Texas portion of the survey.

Two independent intermediate frequencies of $25 \mathrm{MHz}$ bandwidth were centered at 8.415 and $8.465 \mathrm{GHz}$. An onsource dwell time of $30 \mathrm{~s}$ was used. A source from the JVAS

\footnotetext{
5 The National Radio Astronomy Observatory is operated by Associated Universities, Inc., under cooperative agreement with the National Science Foundation.
} 
(Patnaik et al. 1992) was observed after every fourteenth target source for phase calibration. The average target source observation rate was one per minute, including the overhead from calibration and slewing.

The initial calibration of the data was done using AIPS, following the standard procedure. Automatic mapping of the 2575 87GB sources was performed using the DIFMAP package (Shepherd, Pearson, \& Taylor 1994). From the resulting maps $\sim 100$ objects which show multiple components have been selected for further investigation as potential gravitational lens candidates. Only a few of these are expected to be real lensed systems.

The source $1608+656$ (target source $87 \mathrm{~GB} 16087+6540$ ) was observed on 1994 March 1. The VLA image is shown in Figure 1a (Plate L4). The source consists of four wellseparated unresolved components and has a slightly inverted radio spectrum between $325 \mathrm{MHz}$ and $4.85 \mathrm{GHz}$, a property that led to it being followed up with the VLA independently by Snellen et al. among a sample of other inverted-spectrum objects. The results of these and other auxiliary radio observations are described in a companion paper (Snellen et al. 1995).

Several cycles of model fitting and phase-only self-calibration were performed in DIFMAP on the VLA data using four point sources. The residual image showed no missing extended flux. The rms noise level in the final image was $0.44 \mathrm{mJy}$. A total flux density of $73.2 \pm 0.9 \mathrm{mJy}$ was measured. The individual flux densities for components A, B, C, and D were 35.6, 17.8, 15.2, and $4.6 \mathrm{mJy}$, respectively. The brightest component (A) is located at $16^{\mathrm{h}} 09^{\mathrm{m}} 13^{\mathrm{s}} .956,+65^{\circ} 32^{\prime} 28^{\prime \prime} .97$ (J2000). This was 26.4 from the $87 \mathrm{~GB}$ position.

\section{FOLLOW-UP OPTICAL AND INFRARED OBSERVATIONS}

On 1994 August 9, an optical image and spectrum of $1608+656$ were obtained with the Palomar Observatory $5 \mathrm{~m}$ telescope. The optical image (see Fig. 2 [Pl. L6]), taken with the COSMIC camera in 1..5 seeing, is consistent with blended emission from the components in the same configuration as in the radio image as well as extra emission from the presence of a lensing galaxy. Preliminary photometry gives magnitudes in the Gunn system for the entire image (including the lensing galaxy) of $r=19.4 \mathrm{mag}$ and $i=19.2 \mathrm{mag}$. Since the weather was nonphotometric, these may be uncertain by a few tenths of a magnitude.

The spectrum, taken on the same night using the double spectrograph with a 2 " slit accepting most of the light from the aggregate system, is shown in Figure $3 a$. The total exposure was $3000 \mathrm{~s}$. The absorption lines of $\mathrm{Mg}$ II $\lambda \lambda 2796,2803, \mathrm{H} \varepsilon$, $\mathrm{H} \delta$, and the [O II] $\lambda 3727$ emission line are indicated. These yield a redshift of $z=0.6304$ for the lensing galaxy. The Balmer absorption lines are strong, indicating that there are A stars present, and that the lens might possibly be a poststarburst galaxy. The $4000 \AA$ break is weak, which is what would be expected in the combined light of a young stellar population and a background quasar.

On 1994 August 22, a $2.2 \mu \mathrm{m}$ ( $K$-band) infrared image of $1608+656$ (Fig. $1 b$ [Pl. L4]) was obtained on the W. M. Keck ${ }^{6}$ $10 \mathrm{~m}$ telescope using the near-infrared camera (NIRC; Matthews \& Soifer 1994). The total exposure time was $450 \mathrm{~s}$,

${ }^{6}$ The W. M. Keck Observatory is operated as a partnership between the California Institute of Technology and the University of California, and was made possible by the generous gift of the W. M. Keck Foundation.
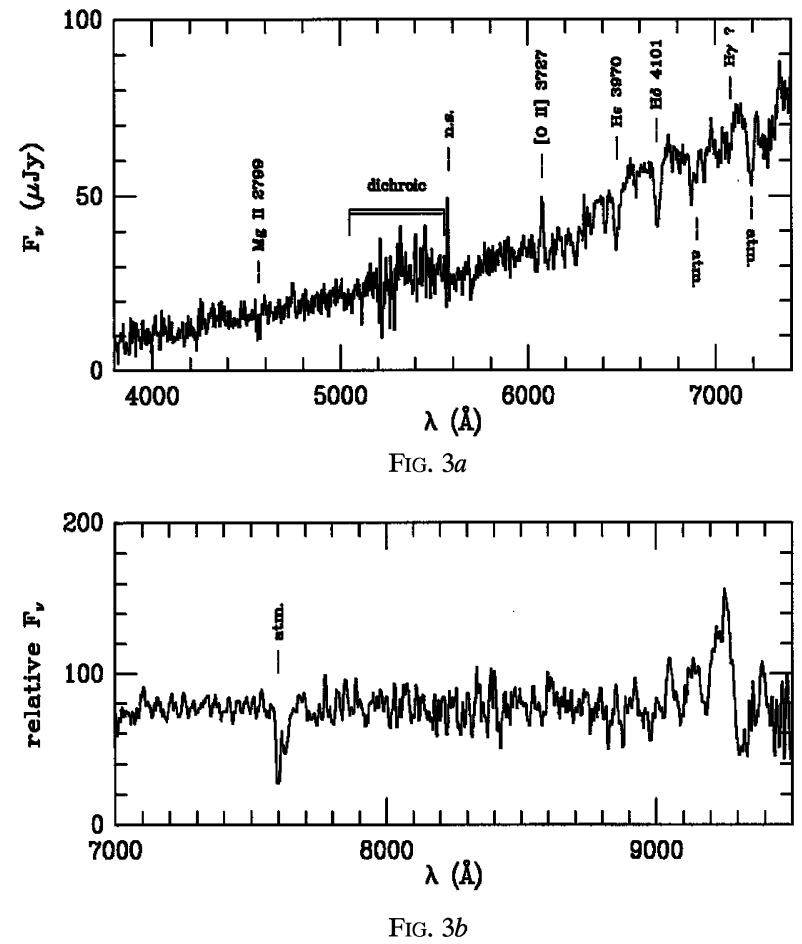

FIG. 3.-(a) Spectrum of $1608+656$ using the double spectrograph at the Palomar $5 \mathrm{~m}$ telescope vs. observed wavelength. The detected emission and absorption features attributed to the lensing galaxy $\left(z_{l}=0.6304\right)$ are indicated. (b) Keck LRIS spectrum, self-normalized to a flat shape since a proper flux calibration is not available. The only convincing feature detected is centered at $9240 \AA ̊$. In both spectra, strong atmospheric absorption (atm.) and night-sky emission (n.s.) features are indicated.

and the seeing was 1 ". The three brightest lens images and the lensing galaxy are seen in this image. A model with four point components was constructed with relative intensities and positions determined from the VLA radio images, convolved with the $K$-band point-spread function, and then subtracted from the NIRC image. The overall scaling factor for the VLA model was adjusted to minimize the residual at the image positions. The results of this are shown in Figure 1c (Plate L5). There is clearly extended emission from the lensing galaxy remaining in the subtracted image. The lensing galaxy has a $K$-band flux roughly 4 times greater than that of the total emission from the four lens components. The galaxy image has an axial ratio of $b / a=0.56 \pm 0.10$ with its major axis in position angle $60^{\circ}$. The true galaxy shape is likely to be more elliptical than this axial ratio would indicate, as the image is substantially circularized by the seeing.

Photometry on the $K$-band residual image yields a mean surface brightness of $60 \mu \mathrm{Jy} \operatorname{arcsec}^{-2}$ within a circle of radius 1".1. This gives a distance-independent mean $K$ surface luminosity, $L_{\mathrm{E}}$, of $7.7 \times 10^{8} L_{\odot} \mathrm{kpc}^{-2}$ for the lensing galaxy. The observed $2.2 \mu \mathrm{m}$ emission was emitted at $1.3 \mu \mathrm{m}$. The ratio of $1.3 \mu \mathrm{m}$ to $V$ fluxes for nearby galaxies is near unity, so the $K$-band luminosity should be roughly equivalent to a $V$ luminosity.

An additional spectrum of the object was obtained using the low-resolution imaging spectrograph (LRIS) on the W. M. Keck 10 m telescope, on the night of UT 1994 September 4, in nonphotometric conditions. The spectrum covers the range 7000-9600 $\AA$, with a resolution of around $7 \AA$ FWHM. 
TABLE 1

PARAMETERS FOR A GRAVITATIONAL Lens MODEL FOR 1608+656

\begin{tabular}{cccccc}
\hline \multicolumn{1}{c}{$\begin{array}{c}\text { Scale Length } \\
r_{c}\end{array}$} & $\begin{array}{c}\text { Mass Parameter } \\
\sigma^{*}\left(\mathrm{~km} \mathrm{~s}^{-1}\right)\end{array}$ & $\begin{array}{c}\text { Eccentricity } \\
e\end{array}$ & $\begin{array}{c}\text { P.A. } \\
\theta\end{array}$ & $\begin{array}{c}\text { Cutoff }^{\mathrm{a}} \\
n\end{array}$ & Lens Center $^{\mathrm{b}}$ \\
\hline $0.48 \ldots \ldots \ldots \ldots \ldots \ldots$ & 311.7 & 0.96 & +67.0 & 20 & $(-0.33,-1.00)$ \\
\hline
\end{tabular}

a The mass model was cutoff at $n$ scale lengths to ensure finite total mass.

$\mathrm{b}$ This value is with respect to image A (see Table 2).

Unfortunately, no standard star exposure was obtained with the same setup, due to instrumental problems. The spectrum is shown in Figure $3 b$, with the baseline artificially flattened. The only nontelluric feature detected is a single strong emission line at $9240 \AA( \pm 50 \AA)$. This line is broader than the instrumental resolution. This does not correspond to any feature expected at the redshift of the lensing galaxy and is assumed to belong to the lensed object.

The plausible identifications for this line are either $\mathrm{Mg}$ II at $z=2.30$ or $\mathrm{H} \beta$ at $z=0.90$. If the $\mathrm{Mg}$ II identification is correct, then Ly $\alpha \lambda 1216$ at $4014 \AA$ and C III] $\lambda 1909$ at $6302 \AA$ would be expected, neither of which is seen in the Palomar spectrum; C IV $\lambda 1549$ at $5113 \AA$ would fall in the dichroic fold of the Palomar data, where the noise is high. The $\mathrm{H} \beta$ interpretation, on the other hand, is supported by the presence of a weak broad feature at $5320 \AA$, which is where Mg II should be found. Unfortunately, this is close to the dichroic fold, and we are not confident of its reality. The $z=0.90 \mathrm{H} \gamma$ line at $8250 \AA$ is not seen in the LRIS spectrum.

\section{A POSSIBLE LENS MODEL}

A lens model for $1608+656$ has been constructed based on the information contained in the VLA $8.4 \mathrm{GHz}$ radio map. An oblate spheroidal mass model has been used for the lens, with mass density $\rho(r)$ following the modified Hubble profile:

$$
\begin{gathered}
\rho(r)=\rho_{0}\left[1+\left(r / r_{c}\right)^{2}\right]^{-3 / 2}, \\
r^{2}=x^{2}+y^{2} /\left(1-e^{2}\right)+z^{2} .
\end{gathered}
$$

The spheroid is assumed to be viewed edge on in order to simplify the model, with $x, y$ in the plane of the sky and $z$ along the line of sight ( $e$ is the eccentricity and $r_{c}$ is the scale length of the mass distribution). Note that the $r^{-3}$ profile assumes that the mass traces the light distribution. The lensing code used was described in Narasimha, Subramanian, \& Chitre (1982, 1984). The constraints are the relative image positions and the relative fluxes, making a total of nine constraints. The model positions were registered on the position of observed component $\mathrm{B}$, which is not so close to the critical surface as A. Fitting of the lens model to the VLA image yields values for the six variable parameters: $r_{c}, e$, a mass parameter $\sigma^{*}$ in units of velocity, the two coordinates of the lens center, and the position angle $\theta$ of the major axis of the spheroid in the plane of the sky.

The set of parameters listed in Table 1 yields a good match to the observations. The position and orientation of the galaxy which is visible in the Keck infrared image agree to within the measurement uncertainties with those predicted by the model. The high eccentricity called for in the mass model for the lens galaxy corresponds to an axial ratio (minor axis to major axis) of $\sqrt{1-e^{2}}=0.28$. The image positions and flux density ratios observed in the VLA image and the predicted model values are listed in Table 2 . The predicted position and magnification for the brightest image A are the most uncertain; therefore, the flux ratios are taken relative to image $\mathrm{B}$. The positions and ratios for images $C$ and $D$ relative to $B$ agree within the measurement uncertainties. The demagnified fifth image $E$ is predicted to be below the detection threshold in the radio map. Even with this preliminary model, the pattern of image positions and flux density ratios is reproduced, strongly suggesting that the basic properties of the lensing potential are well determined.

The lens parameters are cast in a redshift-independent form as in Nair, Narasimha, \& Rao (1993). The expression for the central density in the lens mass distribution is given by:

$$
\rho_{0}=\frac{9 \sigma^{* 2}}{4 \pi G r_{c}^{2}} d_{l s}, \quad d_{l s}=\frac{D_{s}}{D_{l s}}
$$

where $D_{l}, D_{s}$, and $D_{l s}$ are the observer-lens, observer-source, and lens-source angular diameter distances, respectively (e.g., Weinberg 1972). Note that the central velocity dispersion is given by $\sigma=\sigma^{*} \sqrt{d_{l s}}$. For $z_{l}=0.6304$, then, $D_{l}=798 h^{-1} \mathrm{Mpc}$ $\left(q_{0}=0.5\right)$. For the model in Table 1 , we find a central density of $\rho_{0}=4.83 \times 10^{9} d_{l s} h^{2} M_{\odot} \mathrm{kpc}^{-3}$. The mass within the equivalent circular Einstein ring radius $\theta_{\mathrm{E}}=1{ }^{\prime \prime} 1$ is $M_{\mathrm{E}}=1.18 \times 10^{11} d_{l s} h^{-1} M_{\odot}$. The inferred masses and massto-light $\left(K\right.$-band) ratio within $\theta_{\mathrm{E}}$ for $z_{s}=0.90$ and $z_{s}=2.30$,

\begin{tabular}{|c|c|c|c|c|c|c|}
\hline \multirow[b]{2}{*}{ IMAGE } & \multicolumn{2}{|c|}{ OBSERVED } & \multicolumn{4}{|c|}{ MODEL } \\
\hline & Position $^{a}$ & $\begin{array}{l}\text { Flux Ratio } \\
\text { (vs. B) }^{\mathrm{b}}\end{array}$ & Position $^{c}$ & Magnification & $\begin{array}{l}\text { Flux Ratio } \\
\text { (vs. B) }\end{array}$ & $\begin{array}{c}\text { Delay } \\
\left(h^{-1} T_{0} \text { days }\right)\end{array}$ \\
\hline A....... & $(+0.00,+0 " 00)$ & $2.06 \pm 0.06$ & $(+0.15,+0 " 19)$ & +2.58 & 1.68 & 11.9 \\
\hline B....... & $(+0.74,-1.96)$ & 1.00 & $(+0.74,-1.96)$ & +1.54 & 1.00 & 0.0 \\
\hline C $\ldots \ldots$ & $(+0.75,-0.46)$ & $0.85 \pm 0.03$ & $(+0.76,-0.45)$ & -1.36 & 0.88 & 13.1 \\
\hline D...... & $(-1.13,-1.24)$ & $0.26 \pm 0.03$ & $(-1.27,-1.24)$ & -0.35 & 0.23 & 34.2 \\
\hline E...... & $\ldots$ & $\ldots$ & $(-0.52,-1.05)$ & +0.04 & 0.03 & $\ldots$ \\
\hline
\end{tabular}

TABLE 2

IMAGe Properties FOR THE LeNS MODEL

a Position offsets with respect to component A at $16^{\mathrm{h}} 09^{\mathrm{m}} 13 \mathrm{~s} 956,+65^{\circ} 32^{\prime} 28^{\prime \prime} .97$ (J2000).

b Measured $8.4 \mathrm{GHz}$ flux density for component B was $17.80 \pm 0.44 \mathrm{mJy}$.

${ }^{\mathrm{c}}$ Position of model component $\mathrm{B}$ fixed to radio position of image $\mathrm{B}$. 
TABLE 3

Masses and Delays for Possible Source Redshifts

\begin{tabular}{ccccccc}
\hline \hline$z_{s}$ & $d_{l s}$ & $\begin{array}{c}M_{\mathrm{E}} \\
\left(h^{-1} M_{\odot}\right)\end{array}$ & $\begin{array}{c}(M / L)_{\mathrm{E}}{ }^{\mathrm{a}} \\
\left(h M_{\odot} / L_{\odot}\right)\end{array}$ & $T_{0}$ & $\begin{array}{c}\text { C-B Delay } \\
\left(h^{-1} \text { days }\right)\end{array}$ & $\begin{array}{c}\text { C-A Delay } \\
\left(h^{-1} \text { days }\right)\end{array}$ \\
\hline $0.9 \ldots \ldots$ & 4.77 & $5.6 \times 10^{11}$ & 13.2 & 6.19 & 81.1 & 7.67 \\
$2.3 \ldots \ldots$ & 1.93 & $2.3 \times 10^{11}$ & 5.3 & 2.51 & 32.9 & 3.11
\end{tabular}

${ }^{a}$ Using $L_{\mathrm{E}}=7.7 \times 10^{8} L_{\odot} \mathrm{kpc}^{-2}$ from NIRC $K$-band image.

the two most likely source redshifts, are listed in Table 3 . The mass-to-light ratios are similar to those found for other lenses such as MG 1654+1346 (Langston et al. 1990), while the masses are somewhat larger.

The image-plane critical curves, along with the model image positions, and the corresponding source-plane critical curves, with the source position marked, are shown in Figure 4. According to this model, image B should vary first. The predicted time delays are given in Table 2. Here we define $T_{0}=\left(1+z_{l}\right)\left(D_{l} d_{l s} / 1 \mathrm{Gpc}\right)$. The shortest relative pairwise delay is $1.24 T_{0} h^{-1}$ days between images A and C. A FriedmannRobertson-Walker universe with a smoothed-out background matter distribution, $q_{0}=0.5$, and $h=H_{0} / 100 \mathrm{~km} \mathrm{~s}^{-1} \mathrm{Mpc}^{-1}$ is assumed. The predicted relative delays for the source redshifts are given in Table 3. The parameters in our simple lens model, given the missing source redshift, are well constrained by the current observations. VLBI measurements of milliarcsecond component separations in the four images or the discovery of an Einstein ring from lensed jet emission would help to constrain more complicated mass models.
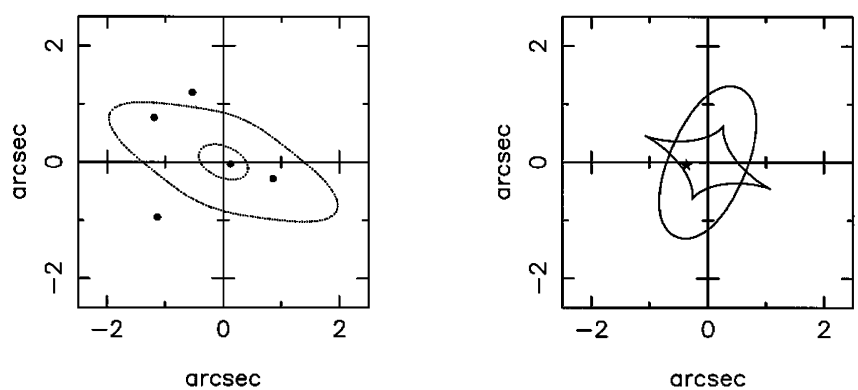

FIG. 4.-Preliminary lens model for the $1608+656$ system. Right: Critical curves in image plane separating domains of 1,3 , and 5 images. Positions of images are marked. Left: Lens mass model in source plane. The images of the inner and outer critical curves are shown. Correspondence of position, ellipticity, and orientation of lensing galaxy model with Keck NIRC residual is good.

\section{DISCUSSION AND CONCLUSIONS}

The necessary ingredients for using a gravitational lens to determine the distance scale are redshifts for the lens and background object, variability in the lensed object with measured time delays between images, and an accurate model for the mass distribution in the lens and along the line of sight to the lens. We have determined the lens redshift and have a tentative redshift for the lensed object. The lensed source is the flat-spectrum core of a double radio source (Snellen et al. 1995), so it is likely that it will be variable at a measureable level. Source variations have allowed measurements of time delays in other systems (e.g., Lehar et al. 1992). The 1608+656 system appears to be simple compared to many other lens systems. The preliminary mass model consisting of a single galaxy with a specified orientation and ellipticity reproduces the observed image and lens configuration. Should further observations support the simple lens model, and should the lens object prove to be variable, $1608+656$ may prove to be an excellent system for determining the Hubble constant.

The lensing galaxy in the $1608+656$ system presents an interesting puzzle. Whether the lens is a single highly elliptical spheroid, a disk galaxy, or a pair of close galaxies has yet to be determined. The large implied mass within the image radius $\left(M_{\mathrm{E}} \sim 5 \times 10^{11} h^{-1} M_{\odot}\right)$ and central velocity dispersion $\left(\sigma \sim 500 \mathrm{~km} \mathrm{~s}^{-1}\right)$ are representative of first-ranked cluster ellipticals, yet the spectrum and high ellipticity are more suggestive of an early-type spiral galaxy. The Balmer absorption is indicative of a poststarburst population of A stars, with the emission line of [O II] caused by ongoing star formation. The large implied ellipticity of the mass distribution, plus the large implied mass, could also be due to a close pair of massive galaxies. The unexpectedly large number of quadruple lenses found in radio surveys, presumably due to highly elliptical potentials in many of the most massive lensing galaxies, may point toward a high merging rate. Hubble Space Telescope observations of this system have been allocated and should provide a much more detailed view of the lensing galaxy.

S. T. M. is supported by an R. A. Millikan Fellowship at Caltech. CLASS at Caltech is supported by NSF grant AST9117100. S. G. D. was supported by NSF PYI award AST9157412. Infrared astrophysics at Caltech is supported by a grant from the NSF. D. S. W. acknowledges support of a Hubble Fellowship provided by NASA through grant HF1040.01-92A from the STScI, which is operated by the AURA. We thank the staff of the VLA and the Palomar and Keck observatories for their assistance during our observing runs. S. T. M. also acknowledges the hospitality of the ITP in Santa Barbara, which is supported by the NSF under grant PHY8904035, during the completion of this manuscript.
Browne, I. W. A., et al. 1995, in preparation de Bruyn, A. G., et al. 1995, in preparation Gregory, P. C., \& Condon, J. J. 1991, ApJS, 75, 1011

Jackson, N., et al. 1995, MNRAS, in press

Langston, G. I., Conner, S. R., Lehar, J., Burke, B. F., \& Weiler, K. W. 1990 Nature, 344, 43

Lehar, J., Hewitt, J. N., Roberts, D. H., \& Burke, B. F. 1992, ApJ, 384, 453

Matthews, K. \& Soifer, T. 1994, in Infrared Astronomy with Arrays: The Next

Generation, ed. I. McLean (Dordrecht: Kluwer), 239

\section{REFERENCES}

Nair, S., Narasimha, D., \& Rao, A. P. 1993, ApJ, 407, 46 Narasimha, D., Subramanian, K., \& Chitre, S. M. 1982, MNRAS, 200, 941 . 1984, MNRAS, 210, 79

Patnaik, A. R., Browne, I. W. A., Wilkinson, P. N., \& Wrobel, J. M. 1992 MNRAS, 254, 655

Shepherd, M. C., Pearson, T. J., \& Taylor, G. B. 1994, BAAS, 26, 987

Snellen, I., et al. 1995, ApJ, 447, L9

Weinberg, S. 1972, Gravitation and Cosmology (New York: Wiley) 
PLATE L4
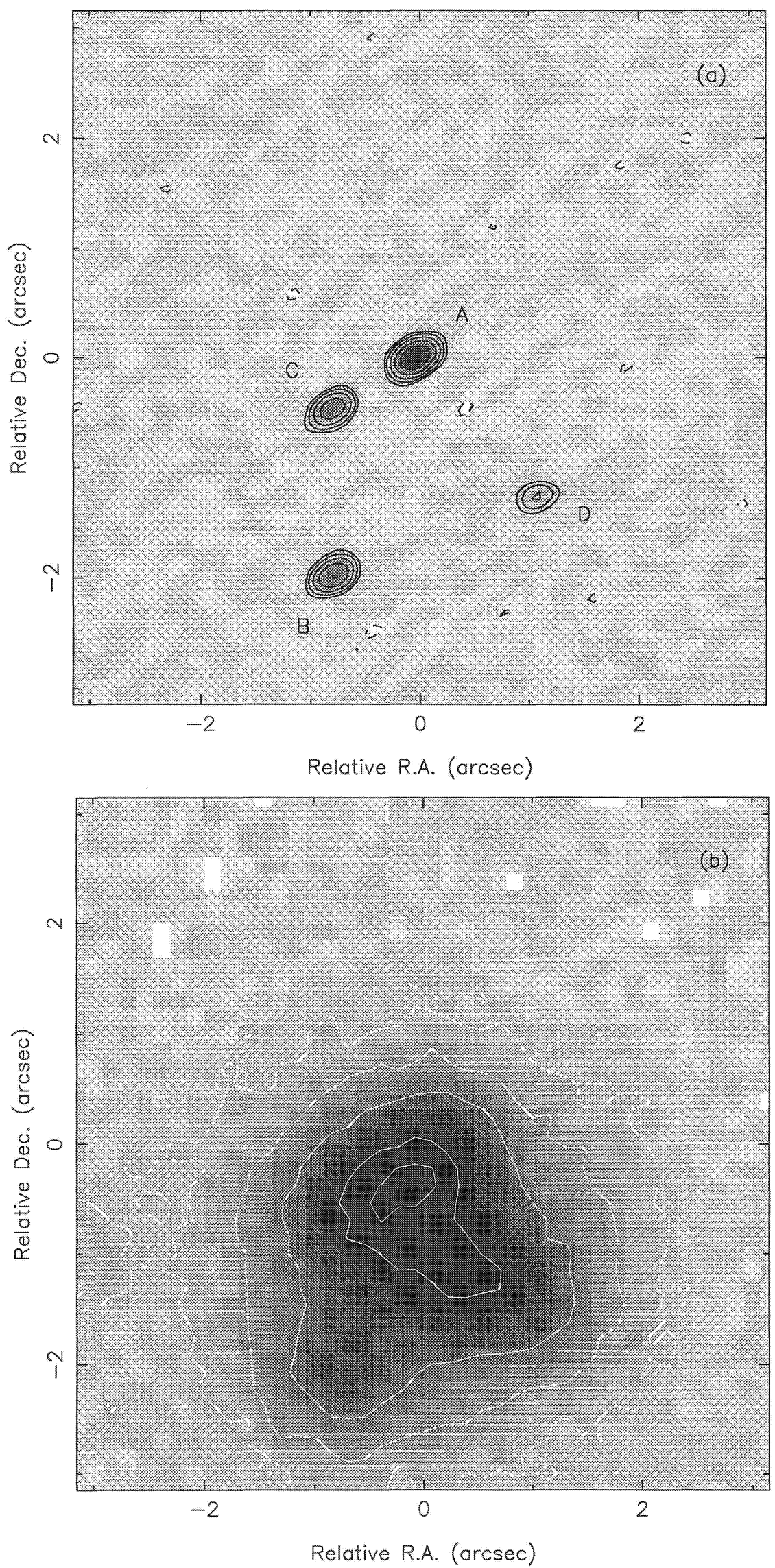

FIG. 1.-CLASS gravitational lens $1608+656$. (a) $8.4 \mathrm{GHz}$ VLA radio image. The peak in the image is $34 \mathrm{mJy}$ beam ${ }^{-1}$. Contours begin at $1.1 \mathrm{mJy}$ beam ${ }^{-1}$ ( 2.5 $\sigma)$, spaced by factors of 2. (b) Keck NIRC image. Seeing is 1". (c) Keck NIRC image with scaled VLA model subtracted. Residual should reflect lensing galaxy emission. Note position, ellipticity, and position angle of galaxy for comparison with the lens model (Fig. 4). 
PLATE L5

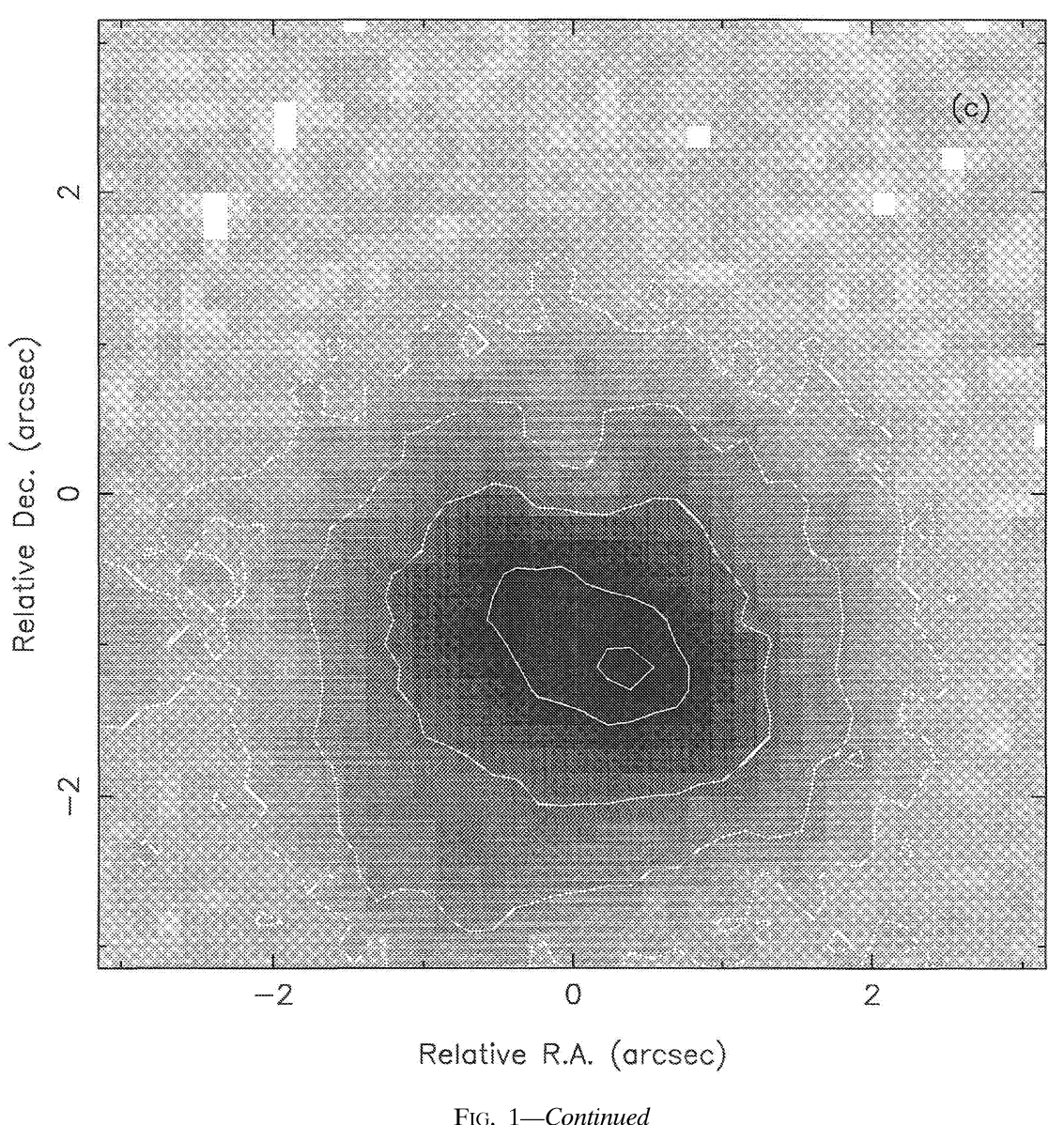

MYERs et al. (see 447, L6) 
PLATE L6

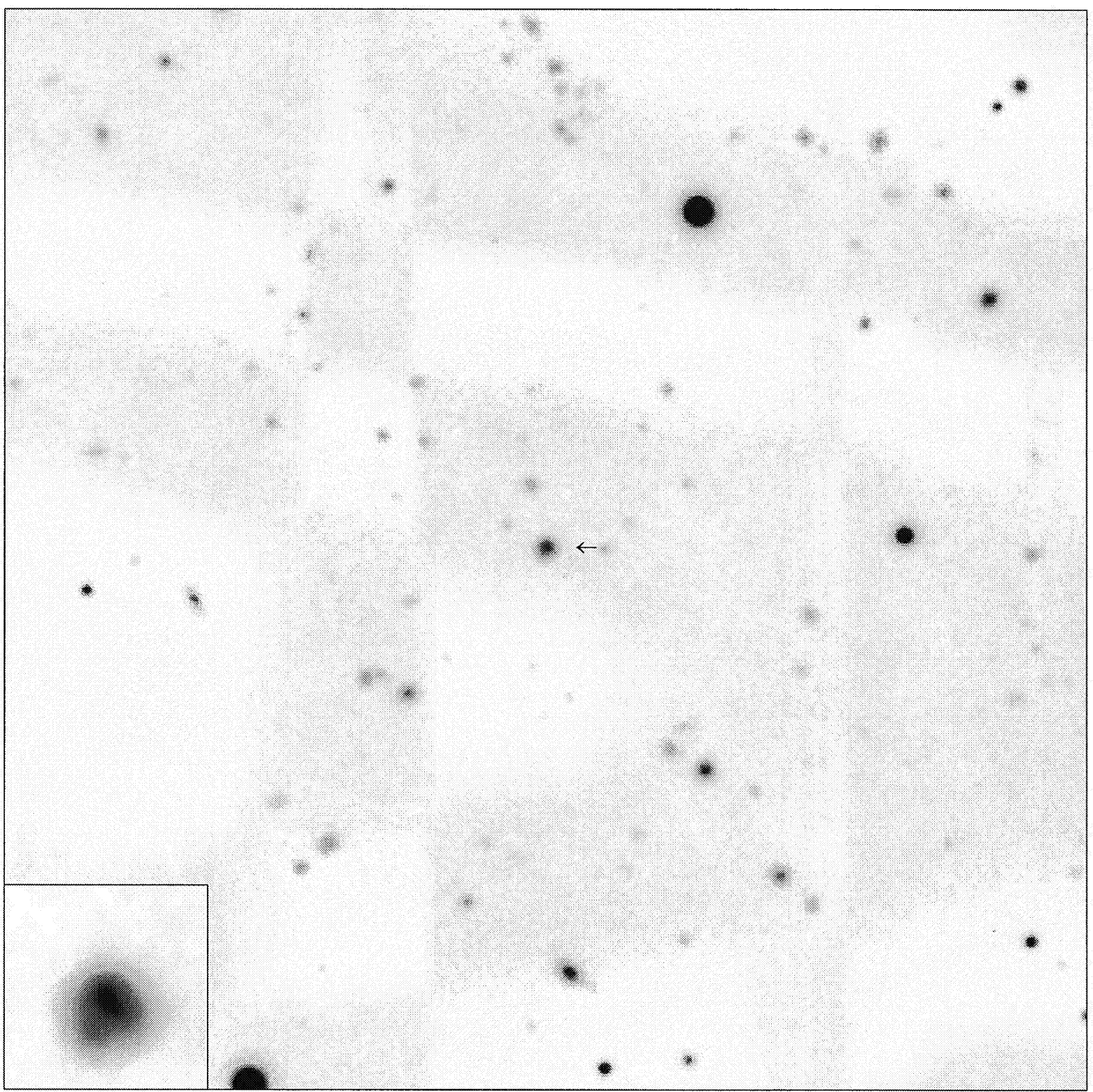

FIG. 2.-An $i$-band image of the field of $1608+656$ obtained at Palomar using COSMIC. The field shown is 200" square, with north at the top and east at the left. The inset image is in the $r$ band and is $10^{\prime \prime}$ square.

MYers et al. (see 447, L6) 